\title{
Pregnancy following treated malignant struma ovarii
}

\author{
IRIIP Ihalagama ${ }^{1}$, S J de S Hewavisenthi ${ }^{2}$ and P S Wijesinghe ${ }^{3}$
}

(Index words: Malignant struma ovarii, pregnancy)

\section{Introduction}

Struma ovarii $[\mathrm{SO}]$ is a rare, highly specialised form of mature ovarian teratoma, consisting predominantly of thyroid tissue. Malignant transformation occurs in about $5 \%$ of SO [1]. Diagnosis of malignant SO is usually made histologically. The majority of malignant SO show a follicular or papillary pattern of thyroid carcinoma. Conservative ovarian surgery is preferred when there is a concern about fertility [2-4].

About 24 cases of malignant SO have been reported until 2003 [5]. Of these only a small number were associated with pregnancy [6-8], in whom diagnosis of malignant SO was made during the pregnancy. Though pregnancy is possible after treatment of malignant $\mathrm{SO}$, no reports are available. We document a rare case of a woman who had two successful pregnancies after treatment of a malignant SO.

\section{Case report}

A 27-year old nulliparous woman was admitted to the gynaecology casualty ward with non-specific lower abdominal pain. Her urinary and bowel habits were normal. There was no change in her body weight or appetite. She had normal menstrual cycles.

On vaginal examination a right adenexal mass of about $10 \mathrm{~cm}$ diameter was detected. It was non-tender and freely mobile. The uterus and the left adenexae were normal. These clinical findings were confirmed by the ultrasound scanning. The right adenexal mass was noted to be an ovarian tumour with solid and cystic areas. The left ovary and the rest of the pelvic cavity were normal. There was no evidence of ascites or hydrothorax. Tumour marker CA 125 was within the normal range.

During laparotomy a right ovarian tumour was identified with evidence of capsular involvement. The left ovary appeared normal. There were no macroscopic deposits in the uterus, tubes, bowels, omentum, liver or undersurface of the diaphragm. As there was evidence of capsular involvement, right salpingo-oophorectomy was performed with a biopsy of the left ovary. Conservative surgery was performed as she wished to preserve her fertility. Histology revealed that the right ovarian tumour was a malignant SO of follicular pattern with foci of vascular invasion. Peritoneal washing was positive for malignant cells. Left ovarian biopsy was normal.

After an uncomplicated immediate post-operative period she was treated with total thyroidectomy and radioactive iodine, followed by daily thyroxine therapy. Within a few months she had a spontaneous conception. After an uncomplicated antenatal period she had a normal delivery. Her thyroid function tests and ultrasound scans of the abdomen were normal. She was pregnant for a second time within 6 months of the first delivery. She had a successful second delivery following an uncomplicated antenatal period.

\section{Discussion}

Struma ovarii is a highly specialised form of monodermal teratoma composed primarily of thyroid tissue [2, 4]. Benign and malignant $\mathrm{SO}$ occur within teratomas at the rate of $2 \%$ and $0.3 \%$ respectively $[2,4]$. Strumal tissue is found contiguous or admixed with carcinoid tumour in strumal carcinoid.

It usually presents with a palpable abdominal mass, occurs more commonly in the left ovary and is sometimes associated with ascites or hydrothorax [2,3,7]. About $10-15 \%$ of patients with SO also have an enlarged thyroid gland [3]. As hyperthyroidism occurs in $8 \%$ of SO, radioiodine imaging of the pelvis should be considered in female hyperthyroid patients [2].

Circulating thyroglobulin receptor antibodies are able to stimulate the ectopic as well as the normal thyroid tissue [3]. Thyroid crisis also may occur rarely after removal of an $\mathrm{SO}[3]$.

The histological criteria for the diagnosis of malignancy in a SO are similar to those of thyroid carcinoma $[2,4]$. However, such an appearance may be mimicked by degenerative changes in either an adenocarcinoma or cystadenocarcinoma [3].

Prognostic indicators for malignant SO include, a diameter greater than $16 \mathrm{~cm}$, ascites and adhesion to neighbouring pelvic structures [2]. Hypercellularity, mitoses, and vascular and capsular invasion are of little prognostic significance in malignant SO [3].

Metastases can be shown in 5-6\% of patients with malignant SO [2]. The mode of spread follows that of an

${ }^{1}$ Registrar in Obstetrics and Gynaecology, Teaching Hospital, Ragama; ${ }^{2}$ Department of Pathology, ${ }^{3}$ Department of Obstetrics and Gynaecology, Faculty of Medicine, Ragama, Sri Lanka.

Correspondence: PSW, e-mail: <pswijesinghe@lycos.com> (Competing interests: none declared). Received 1 March 2004 and accepted 19 April 2004. 
ovarian carcinoma. Peritoneal dissemination can simulate benign strumosis, a peritoneal seeding of benign $\mathrm{SO}$, and is composed of mature thyroid tissue [2].

Because of the rarity of malignant $\mathrm{SO}$, the optimal surgical and post-operative management has not been defined $[1,2]$. Therapy has ranged from radical surgery and chemotherapy, with or without thyroidectomy, to unilateral oophorectomy and preservation of reproductive functions [2]. In patients with residual abdominal disease or with distant metastases, total thyroidectomy followed by the administration of radioactive iodine should be offered [1,2]. Prognosis as well as survival improves with the administration of radioactive iodine $[1,2]$. In one study, no deaths were reported in eight patients who received radioactive iodine for malignant $\mathrm{SO}$ with adequate follow up of 7.3 years [2]. Our patient had a good prognosis in spite of having peritoneal metastases. This suggests that the peritoneal metastases would have been effectively treated by radioactive iodine. Prognosis can be monitored using thyroglobulin levels and radioactive scintigraphy $[1,4]$.

Pregnancy is possible after conservative surgery for malignant SO. A few case reports have been published on malignant SO found during pregnancy [6-8]. None of them, however, described successful pregnancy after treatment of malignant SO.

\section{References}

1. Rose PG, Arafah B, Abdul-Karim FW. Malignant struma ovarii: recurrence and response to treatment monitored by thyroglobulin levels. Gynecologic Oncology 1998; 70: 425-7.

2. Dardik RB, Dardik M, Westra W, Montz FJ. Malignant struma ovarii: two case reports and a review of the literature. Gynecologic Oncology 1999; 73: 447-51.

3. Fox H, Wells M H. Taylor Obstetrical and Gynaecological Pathology. New York: Churchill Livingstone, 1995.

4. Vadmal MS, Smilari TF, Lovecchio JL, Klein IL, Hajdu SI. Diagnosis and treatment of disseminated struma ovarii with malignant transformation. Gynecologic Oncology 1997; 64: 541-6.

5. De Simone CP, Lele SM, Modesitt SC. Malignant struma ovarii: a case report and analysis of cases reported in the literature with focus on survival and $\mathrm{I}^{131}$ therapy. Gynecologic Oncology 2003; 89: 543-8.

6. Tokuda Y, Hatayama T, Sakoda K. Metastasis of malignant struma ovarii to the cranial vault during pregnancy. Neurosurgery 1993; 33: 515-8.

7. Kung AW, Ma JT, Wang C,Young RT. Hyperthyroidism during pregnancy due to coexistence of struma ovarii and Graves' disease. Postgraduate Medical Journal 1990; 66: 132-3.

8. Moggian G, Saragoni A, Pellegri E, Tamburili E, Pini PL, et al. Struma ovarii: report of a case with malignant evolution and of another observed in the course of pregnancy. European Journal of Gynecologic Oncology 1980; 1: 201-2 